\title{
Critical Ising lines of the $d=2$ Ashkin-Teller model
}

\author{
G. Kamieniarz* and P. Kozłowski* \\ Computational Physics Division, Institute of Physics, A. Mickiewicz University, ul. Umultowska 85, PL 61-614 Poznań, Poland

\section{R. Dekeyser} \\ Institute for Theoretical Physics, Katholieke Universiteit Leuven, Celestijnenlaan 200D, B 3001 Leuven, Belgium
}

(Received 22 October 1996)

\begin{abstract}
The universal critical point ratio $Q$ is exploited to determine positions of the critical Ising transition lines on the phase diagram of the Ashkin-Teller model on the square lattice. A leading-order expansion of the ratio $Q$ in the presence of a nonvanishing thermal field is found from finite-size scaling and the corresponding expression is fitted by the accurate perturbative transfer-matrix data calculations for the $L \times L$ square clusters with $L \leqslant 9$. [S1063-651X(97)04503-0]
\end{abstract}

PACS number(s): 05.50.+q, 05.70.Jk, 64.60.Fr

The Ashkin-Teller (AT) model has first been proposed as a model of a four component alloy [1]. It has attracted a lot of theoretical interest for years because it is a simple and nontrivial generalization of the Ising and four-state Potts models. Fan [2] has shown that the Hamiltonian of the AT model can also be written with two Ising variables ( $S= \pm 1, \sigma= \pm 1$ ) located at each site of the lattice, which in the presence of a magnetic field has the form

$$
\mathcal{H}=-\sum_{\langle i, j\rangle}\left(J_{1} S_{i} S_{j}+J_{2} \sigma_{i} \sigma_{j}+J_{4} S_{i} \sigma_{i} S_{j} \sigma_{j}+J_{0}\right)-h \sum_{i=1}^{N} S_{i} \sigma_{i} .
$$

Herein we consider only the nearest neighbor pair interactions on the simple square lattice consisting of $N=L^{2}$ sites with periodic boundary conditions and we assume that $J_{1}=J_{2}$ (isotropic case).

Wagner [3] has shown that the AT model is equivalent to the alternated eight vertex model, which has not been solved exactly. Only one critical line in the phase diagram of the isotropic AT model is known exactly thanks to the duality relation found by Fan [4]. For this reason many approximate approaches have been applied for constructing the complete phase diagram: the mean-field approximation (MFA) $[5,6]$, mean-field renormalization group (MFRG) [7], renormalization group (RG) [8], and Monte Carlo renormalization group (MCRG) [9]. It is the aim of this paper to establish an accurate location of the remaining critical lines.

In our approach we exploit finite-size scaling for the ratio of the square of the second moment to the fourth moment of the order parameter $M$ :

$$
Q_{L}=\frac{\left\langle M^{2}\right\rangle_{L}^{2}}{\left\langle M^{4}\right\rangle_{L}}
$$

where $\langle\ldots\rangle$ means thermal average and the index $L$ indicates the linear size of the system $(L \times L)$. In the limit $L \rightarrow \infty$ this ratio becomes universal in the critical point [10]

\footnotetext{
*Electronic address: gjk@ pearl.amu.edu.pl

†Electronic address: raf.dekeyser@fys.kuleuven.ac.be
}

and is denoted $Q$ hereafter. Three not exactly known critical lines of the isotropic AT model are believed to belong to the Ising universality class $[5,11]$. Here it is assumed that these lines correspond to the Ising-like continuous transitions with the order parameter $M=\sum_{i=1}^{N} S_{i} \sigma_{i}$. A scaling formula for $Q_{L}$ can be derived starting from the finite-size scaling relation for the singular part of the free energy for the square Ising model [12].

$$
F^{(S)}\left(g_{t}, g_{h}, L^{-1}\right)=A\left(g_{t} L\right) \ln L+B\left(g_{t} L, g_{h} L^{y_{h}}\right),
$$

where $A$ and $B$ are unknown amplitudes, $g_{t}, g_{h}$ are nonlinear scaling fields, and $y_{h}$ is the magnetic critical exponent. The nonlinear scaling fields $g_{t}$ and $g_{h}$ can be expanded in terms of the corresponding linear thermal and magnetic scaling fields $t$ and $h$.

Taking into account the relations between the magnetization moments in Eq. (2) and the corresponding derivatives of the free energy [12] we have calculated the scaling expansion for $Q_{L}(t, h=0)$ to the leading order in $t$ and up to $L^{3-4 y_{h}}$ :

$$
Q_{L}(t)=Q_{L}(0)+\left.\frac{\partial Q_{L}(t)}{\partial t}\right|_{t=0} t+\cdots
$$

The zeroth-order term $Q_{L}(0)$ was evaluated previously [12] and the first-order term is of the form

$$
\begin{aligned}
\left.\frac{\partial Q_{L}(t)}{\partial t}\right|_{t=0}= & \alpha_{1} L+\alpha_{2}+\alpha_{3} L^{3-2 y_{h}}+\left(\alpha_{4}+\alpha_{5} \ln L\right) L^{2-2 y_{h}} \\
& +\alpha_{6} L^{5-4 y_{h}}+\left(\alpha_{7}+\alpha_{8} \ln L\right) L^{1-2 y_{h}} \\
& +\left(\alpha_{9}+\alpha_{10} \ln L\right) L^{4-4 y_{h}}+\alpha_{11} L^{-2 y_{h}} \\
& +\left(\alpha_{12}+\alpha_{13} \ln L\right) L^{7-6 y_{h}} \\
& +\left(\alpha_{14}+\alpha_{15} \ln L+\alpha_{16} \ln ^{2} L\right) L^{3-4 y_{h}}+\cdots
\end{aligned}
$$

where $\alpha_{i}(i=1, \ldots, 16)$ are unknown amplitudes. In our work we consider only the first three terms in the expansion (5), but for some future Monte Carlo applications the higherorder terms in $1 / L$ might be important. 


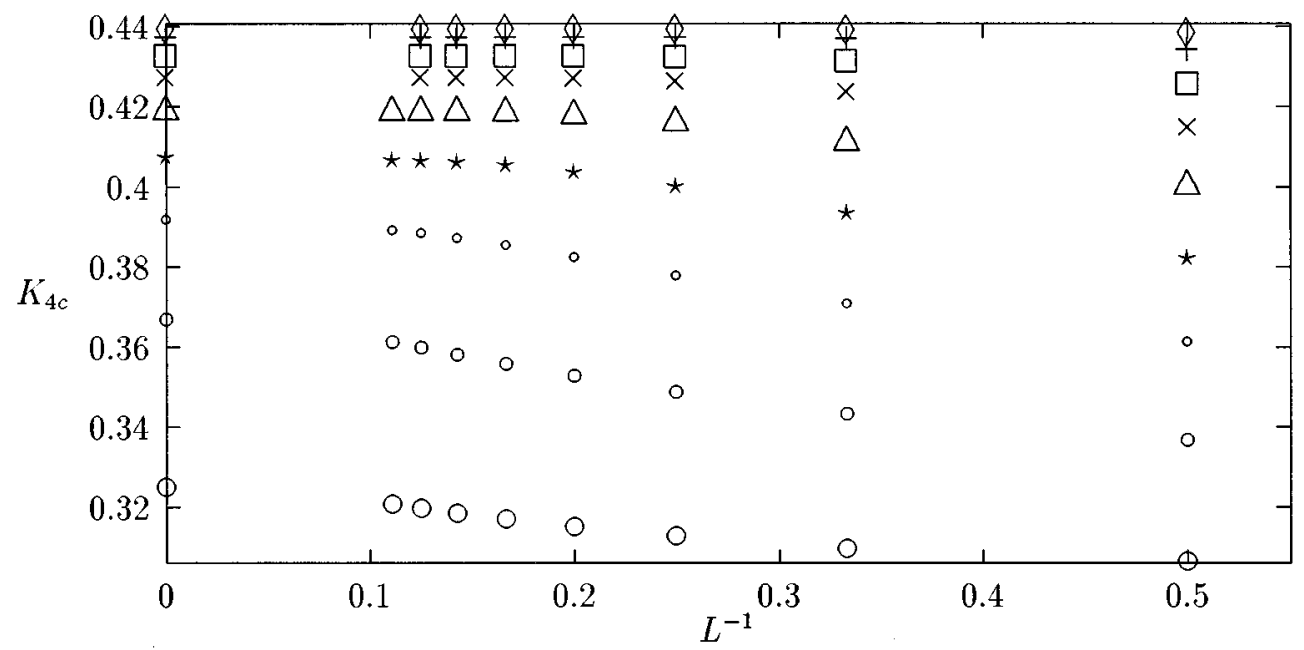

FIG. 1. The $L$ dependence of the critical values of the parameter $K_{4 c}$. The points on the vertical axis are the extrapolated values.

We have calculated the $Q_{L}(t)$ ratio exploiting the transfer-matrix technique which for the Ising model was explained in [12]. Our system consists of $L$ columns containing $L$ sites. Spins from the $j$ th column are denoted by $\vec{\Sigma}_{j}=\left(S_{j 1}, \sigma_{j 1}, S_{j 2}, \sigma_{j 2}, \ldots, S_{j L}, \sigma_{j L}\right)$ so that

$$
Z=\sum_{\vec{\Sigma}_{1}, \vec{\Sigma}_{2}, \ldots, \vec{\Sigma}_{L}} \exp \left[-\beta H\left(\vec{\Sigma}_{1}, \ldots, \vec{\Sigma}_{L}\right)\right]=\operatorname{Tr}^{L}
$$

where $\mathbf{T}$ is a $4^{L} \times 4^{L}$ transfer matrix. This can be split into the product $\mathbf{T}=\mathbf{T}_{h} \mathbf{T}_{v}$ of a diagonal matrix $\mathbf{T}_{v}$ and a nondiagonal matrix $\mathbf{T}_{h}$ containing the intracolumn and the intercolumn interactions, respectively. They are defined as follows:

$$
\begin{gathered}
\mathbf{T}_{v}\left(\vec{\Sigma}_{k}, \vec{\Sigma}_{l}\right)=\delta_{\vec{\Sigma}_{k}, \vec{\Sigma}_{l}} \exp \left(\sum _ { i = 1 } ^ { N } \left(K_{2} S_{k, i} S_{k, i+1}+K_{2} \sigma_{k, i} \sigma_{k, i+1}\right.\right. \\
\left.\left.+K_{4} S_{k, i} \sigma_{k, i} S_{k, i+1} \sigma_{k, i+1}+H S_{k, i} \sigma_{k, i}\right)\right) \\
\mathbf{T}_{h}\left(\vec{\Sigma}_{k}, \vec{\Sigma}_{l}\right)=\exp \left(\sum _ { i = 1 } ^ { N } \left(K_{2} S_{k, i} S_{l, i}+K_{2} \sigma_{k, i} \sigma_{l, i}\right.\right. \\
\left.\left.+K_{4} S_{k, i} \sigma_{k, i} S_{l, i} \sigma_{l, i}\right)\right)
\end{gathered}
$$

where $\beta=1 / k_{B} T, K_{i}=J_{i} \beta(i=1,2,4)$, and $H=\beta h$. The latter matrix can be expressed as a product of sparse matrices, which facilitates the numerical calculations.

The averages in Eq. (2) can be expressed in terms of the corresponding coefficients $Z_{k}$ [12] in the expansion of the field dependent partition function $Z(h)=\sum_{k=0}^{\infty} Z_{k}\left(h^{k} / k !\right)$. The coefficients $Z_{k}$ can then be calculated from Eq. (6) by multiplying the base vectors by matrices $\mathbf{T}_{v}$ and $\mathbf{T}_{h}$ in such a manner that the terms in the same power of $h$ are kept separately [12].

At first we calculate the amplitudes $\alpha_{i}(i \leqslant 5)$ from Eqs. (4) and (5) with known values $Q_{L}(0)$. In the limit $K_{2}=0$, i.e., the Ising model in $S \sigma, K_{4 c}=K_{c}=\frac{1}{2} \ln (1+\sqrt{2})$ and in this case we have only one coupling constant $\left(K_{4}\right)$. Thus we can write the reduced temperature in the form

$$
t=\frac{K_{4 c}-K_{4}}{K_{4}}
$$

Selecting different values of the scaling field $t$ we can solve the set of linear algebraic equations for $\alpha_{i}$. For the ferromagnetic coupling $K_{4}$ we consider the system sizes $L=2,3, \ldots, 9$ whereas for the antiferromagnetic one only the even values $L=2,4,6,8$ are considered, so that we can evaluate the coefficients $\alpha_{i}$ up to $i=5$ or $i=3$, respectively.

Having fixed $K_{2} \neq 0$ and knowing the $\alpha_{i}(i \leqslant 3)$ and $Q_{L}(0)$, we have calculated $Q_{L}\left(K_{2}, K_{4}\right)$ for a number of couplings $K_{4}$. This enables a determination of the corresponding $t$ values from Eqs. (4) and (5). Then knowing $t$ we can easily obtain $K_{4 c}$ from Eq. (9) and $K_{2 c}$ from a similar equation, but written for $K_{2}$. The estimates $K_{4 c}$ and $K_{2 c}$ are very stable if we find $t \in\left\langle 10^{-7}, 10^{-4}\right\rangle$.

The exactly known critical curve with continuously varying critical exponents [11] is terminated in the four-state Potts point where it bifurcates. In the vicinity of this point the convergence of our results is diminished and the estimates of $K_{4 c}$ become size dependent. This size dependence is illustrated in Fig. 1. Due to the limited number of system sizes available in our calculations we do not try to include any corrections to scaling and we simply extrapolate our data. The corresponding estimates are shown on the ordinate axis in Fig. 1. Such a strong size dependence does not occur for the antiferromagnetic couplings, since there is no Potts point in this case.

Our final results represented by open circles connected by thin continuous lines are shown in Fig. 2 and they are compared with other results and predictions. The numerical uncertainties do not exceed the size of the symbol. The curve plotted by the bold line represents the part of the phase diagram found exactly by Baxter [11]. It separates the Baxter phase $B$ from the paramagnetic phase $P$. The ferromagnetic and antiferromagentic phases with nonvanishing order parameter $M$ are denoted by the labels $F$ and $A F$, respectively. 


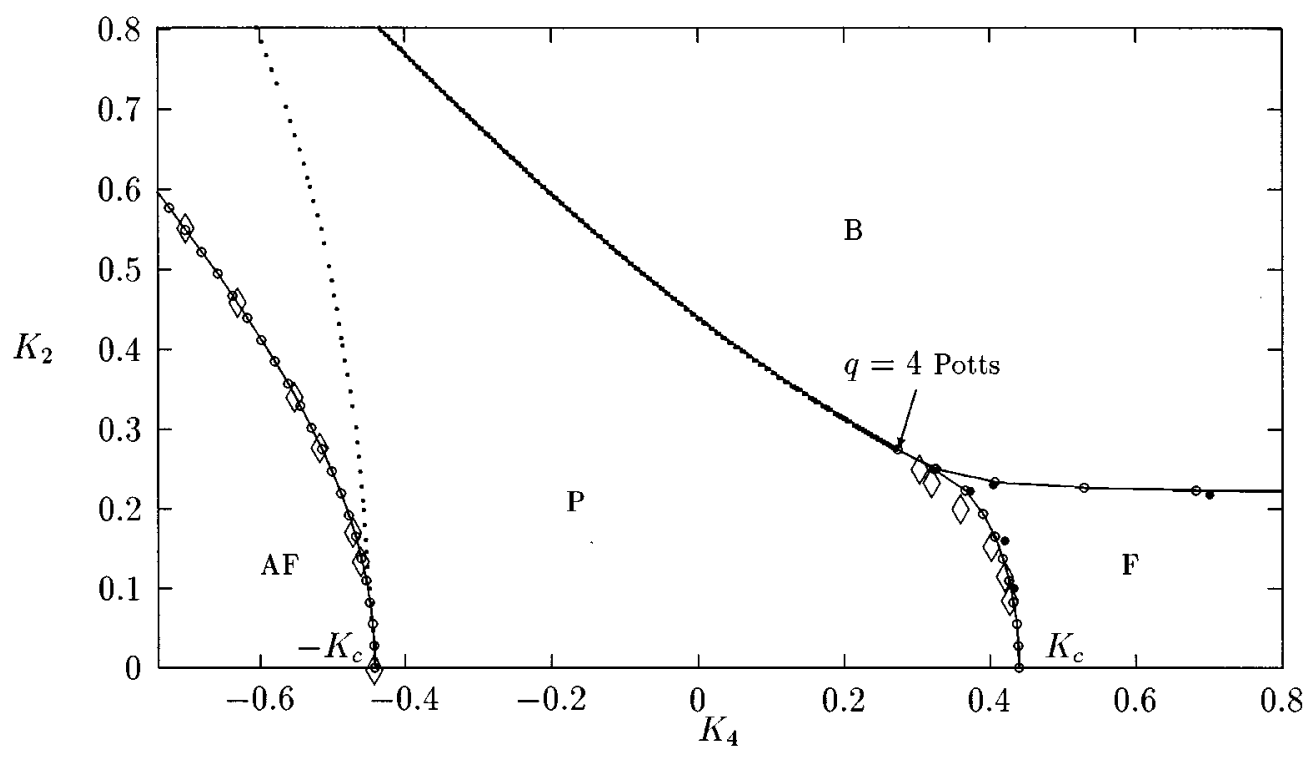

FIG. 2. Phase diagram of the AT model in two dimensions. The solid bold line represents the exactly known critical line, which terminates at the four-state Potts point. Empty circles with continuous lines describe our results. The solid circles display MCRG results, dotted line is drawn after Baxter, and diamonds are the transfer-matrix results combined with conformal invariance.

In the ferromagnetic region $K_{4}>0$ we have only calculated the curve joining the four-state Potts point to the pure Ising point $K_{c}$ at $K_{2}=0$. The second branch follows from the corresponding duality relation $[5,11]$. In the boundary between $A F$ and $P$ phases with the dotted lines we plot the approximate curve as given by Baxter [11] and in the ferromagnetic region we also include the MCRG results marked by filled circles.

As can be seen (Fig. 2) our results are in good agreement with the MCRG [9] approach, but are quite different from Baxter's predictions [11] in the antiferromagnetic region. For the boundary between $A F$ and $P$ phases, our results coincide with those obtained by Mazzeo et al. [13]. These authors actually investigated the six vertex model with the transfermatrix technique in combination with conformal invariance arguments; their results can be mapped onto the results for the $P$-phase boundaries and they are shown in Fig. 2.

As to our accuracy: near the ferromagnetic Ising point it is of about $2 \times 10^{-6}$ and in the neighborhood of the Potts point it decreases down to about $3 \times 10^{-2}$. The accuracy in the antiferromagnetic region is even better: near the Ising point it reaches $5 \times 10^{-8}$ and for the highest point at the phase diagram in Fig. 2 it decreases to $3 \times 10^{-3}$.

The numerical calculations were carried out in the Supercomputing and Networking Center in Poznan on the Cray J-916. The work has been supported in part by the Committee for Scientific Research via Grant No. 2 P302 11606 . We also thank Dr. E. Carlon, Dr. P. Pawlicki, and Professor J. Rogiers for some discussions.
[1] J. Ashkin and E. Teller, Phys. Rev. 64, 178 (1943).

[2] C. Fan, Phys. Lett. 39A, 136 (1972).

[3] F. J. Wagner, J. Phys. C 5, L131 (1972).

[4] C. Fan, Phys. Rev. B 6, 902 (1972).

[5] R. V. Ditzian, J. R. Banavar, G. S. Grest, and L. P. Kadanoff, Phys. Rev. B 22, 2542 (1980).

[6] P. Pawlicki and J. Rogiers, Physica A 214, 277 (1995).

[7] J. A. Plascak and F. C. Sá Barreto, J. Phys. A 19, 2195 (1986).

[8] N. Benayad, A. Benyoussef, N. Boccara, and A. El. Kenz, J. Phys. C 21, 5747 (1988).
[9] J. Chahine, J. R. Drugowich de Felicio, and N. Caticha, J. Phys. A 22, 1639 (1989).

[10] P. C. Privman, P. C. Hohenberg, and A. Aharony, in Phase Transition and Critical Phenomena, edited by C. Domb and J. L. Lebowitz (Academic Press, New York, 1991), Vol. 14.

[11] R. J. Baxter, Exactly Solved Models in Statistical Mechanics (Academic, New York, 1982).

[12] G. Kamieniarz and H. W. J. Blöte, J. Phys. A 26, 201 (1993).

[13] G. Mazzeo, E. Carlon, and H. van Beijeren, Phys. Rev. Lett. 74, 1391 (1995). 